\title{
RESEARCH
}

Open Access

\section{Cholangiogenic potential of human deciduous pulp stem cell-converted hepatocyte-like cells}

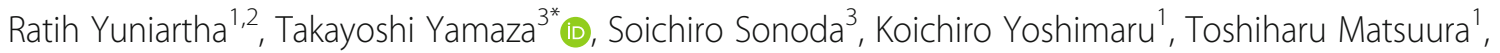
Haruyoshi Yamaza ${ }^{4}$, Yoshinao Oda ${ }^{5}$, Shouichi Ohga ${ }^{6}$ and Tomoaki Taguchi ${ }^{1,7}$

\begin{abstract}
Background: Stem cells from human exfoliated deciduous teeth (SHED) have been reported to show the in vivo and in vitro hepatic differentiation, SHED-Heps; however, the cholangiogenic potency of SHED-Heps remains unclear. Here, we hypothesized that SHED-Heps contribute to the regeneration of intrahepatic bile duct system in chronic fibrotic liver.

Methods: SHED were induced into SHED-Heps under cytokine stimulation. SHED-Heps were intrasplenically transplanted into chronically $\mathrm{CCl}_{4}$-treated liver fibrosis model mice, followed by the analysis of donor integration and hepatobiliary metabolism in vivo. Immunohistochemical assay was examined for the regeneration of intrahepatic bile duct system in the recipient liver. Furthermore, SHED-Heps were induced under the stimulation of tumor necrosis factor alpha (TNFA).

Results: The intrasplenic transplantation of SHED-Heps into $\mathrm{CCl}_{4}$-treated mice showed that donor SHED-Heps behaved as human hepatocyte paraffin 1- and human albumin-expressing hepatocyte-like cells in situ and ameliorated $\mathrm{CCl}_{4}$-induced liver fibrosis. Of interest, the integrated SHED-Heps not only expressed biliary canaliculi ATP-binding cassette transporters including $A B C B 1, A B C B 11$, and $A B C C 2$, but also recruited human keratin 19(KRT19-) and KRT17-positive cells, which are considered donor-derived cholangiocytes, regenerating the intrahepatic bile duct system in the recipient liver. Furthermore, the stimulation of TNFA induced SHED-Heps into KRT7- and SRY-box 9-positive cells.

Conclusions: Collectively, our findings demonstrate that infused SHED-Heps showed cholangiogenic ability under the stimulation of TNFA in $\mathrm{CCl}_{4}$-damaged livers, resulting in the regeneration of biliary canaliculi and interlobular bile ducts in chronic fibrotic liver. Thus, the present findings suggest that SHED-Heps may be a novel source for the treatment of cholangiopathy.
\end{abstract}

Keywords: Human deciduous pulp stem cell-converted hepatocyte-like cells, Intrahepatic bile duct regeneration, Cholangiocyte, Tumor necrosis factor alpha, Chronic liver fibrosis

\footnotetext{
* Correspondence: yamazata@dent.kyushu-u.ac.jp

${ }^{3}$ Department of Molecular Cell Biology and Oral Anatomy, Kyushu University

Graduate School of Dental Science, 3-1-1 Maidashi, Higashi-ku, Fukuoka

812-8582, Japan

Full list of author information is available at the end of the article
}

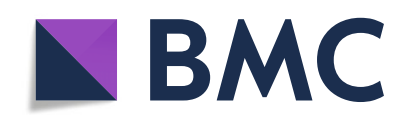

(- The Author(s). 2021 Open Access This article is licensed under a Creative Commons Attribution 4.0 International License, which permits use, sharing, adaptation, distribution and reproduction in any medium or format, as long as you give appropriate credit to the original author(s) and the source, provide a link to the Creative Commons licence, and indicate if changes were made. The images or other third party material in this article are included in the article's Creative Commons licence, unless indicated otherwise in a credit line to the material. If material is not included in the article's Creative Commons licence and your intended use is not permitted by statutory regulation or exceeds the permitted use, you will need to obtain permission directly from the copyright holder. To view a copy of this licence, visit http://creativecommons.org/licenses/by/4.0/ The Creative Commons Public Domain Dedication waiver (http://creativecommons.org/publicdomain/zero/1.0/) applies to the data made available in this article, unless otherwise stated in a credit line to the data. 


\section{Background}

Orthotopic liver transplantation (OLT) is the only option to ameliorate liver refractory diseases, such as chronic liver fibrosis [1]. However, approximately $10 \%$ of patients with end-staged hepatic disorders are not able to receive the benefit owing to the extended waiting period and the shortage of donor organs [2, 3]. Human primary hepatocyte transplantation (hPHepT) becomes a considerable alternative to OLT, especially in liver-based inborn errors of hepatic metabolism and acute/fulminant liver failure [4-6]. Despite the clinical advantages of hPHepT, such as a less-invasive procedure, a current clinical limitation of clinical hPHepT is set by the shortage of effective donor hepatocytes and temporal efficacy [7].

Diverse human mesenchymal stem/stromal cell (MSC)-derived hepatocyte-like cells (HLCs) have been investigated as alternatives to hPHeps $[8,9]$. Human deciduous pulp stem cells were first identified in the dental pulp tissues of exfoliated deciduous teeth, namely stem cells from human exfoliated deciduous teeth (SHED) [10], and are beneficial for the treatment of autoimmune, liver, and spinal injury diseases [11-13]. Recent studies demonstrated that SHED exhibit a hepatic potency under a sequential stimulation of hepatogenic cytokines, as referred to SHED-Heps, but SHED-Heps were immature and showed limited-function as hepatocytes $[14,15]$.

Chronic injury often causes ductular reaction in liver $[16,17]$. Liver fibrosis is closely associated with the ductular reaction, resulting in bile excretion damage [18]. However, which therapeutic option regenerates intrahepatic bile ducts in cholestasis associated with liver diseases has yet not been elucidated. In this study, we investigated whether donor SHED-Heps recruited intrahepatic bile duct system, as well as reconstructed parenchymal hepatocytes, in fibrotic liver of chronically $\mathrm{CCl}_{4}$ treated mice. Further, we also investigated if SHEDHeps could be induced into cholangiocyte markerexpressing cells cultured under tumor necrosis factor alpha (TNFA) stimulation. Thus, this study aims to demonstrate our hypothesis of the cholangiogenic potency in vivo of SHED-Heps.

\section{Methods}

\section{Mice}

C57BL/6J mice (female, 6-week old) were purchased from Charles River Laboratories Japan (Yokohama, Japan). All animal experiments were approved by the Institutional Animal Care and Use Committee of Kyushu University (approval no. A21-044-1 and A20-041-0). All animals were housed in temperature- and lightcontrolled conditions with a 12-h light and dark cycle, and fed water and standard pellet chow ad libitum.

\section{Antibodies}

Additional file 1 Supplementary Table 1 lists the antibodies used in this study.

\section{Isolating, culturing, and characterizing SHED and generating SHED-Heps}

SHED were isolated, cultured, and characterized according to previous studies $[10,11]$ (Additional file 1: Supplementary Fig. 1). The details of SHED culture procedures are as described in the Additional file 1: Supplementary Methods.

\section{Induction of SHED-Heps}

P3 SHED were seeded at $2.5 \times 10^{5}$ cells per dish on human fibronectin-coated 100-mm culture dishes (Corning) and maintained in a growth medium. After they reached confluency, SHED were treated with epidermal growth factor (EGF; $20 \mathrm{ng} / \mathrm{mL}$; PeproTech, Rocky Hill, NJ) and fibroblast growth factor 2 (FGF2; $10 \mathrm{ng} / \mathrm{mL}$; PeproTech) in Iscove's modified Dulbecco's media (IMDM; Thermo Fisher Scientific, Waltham, MA) and premixed antibiotics of $100 \mathrm{U} / \mathrm{ml}$ penicillin and $100 \mu \mathrm{g} /$ $\mathrm{ml}$ streptomycin (premixed P/S antibiotics; Nacalai Tesque, Kyoto, Japan) for 2 days [14, 19] (Supplementary Fig. S2a). Subsequently, the cells were cultured using hepatogenic cytokines and regents. Initially, the cells were stimulated with FGF2 (10 ng/mL; PeproTech), hepatocyte growth factor (HGF; $20 \mathrm{ng} / \mathrm{mL}$; PeproTech), and nicotinamide ( $0.61 \mathrm{~g} / \mathrm{L}$; Merck, Darmstadt, Germany) in IMDM (Thermo Fisher Scientific) and premixed P/S antibiotics (Nacalai Tesque) for 7 days. Finally, they were stimulated with oncostatin M (20 ng/mL; PeproTech), dexamethasone $(1 \mu \mathrm{M} ;$ Merck), insulin-transferrinselenium premix solution (ITS) premix (1×; Corning), and premixed $\mathrm{P} / \mathrm{S}$ antibiotics (Nacalai Tesque) for 21 days. Each medium was changed twice weekly. The generated SHED-Heps were re-seeded at $0.1 \times 10^{6}$ per well in low cell attachment PrimeSurface $96 \mathrm{U}$ multiwell plates (Sumitomo Bakelite, Tokyo, Japan). The medium contained oncostatin M (20 ng/mL; Pepro Tech), dexamethasone ( $1 \mathrm{mM}$; Merck), ITS premix (1×; Thermo Fisher Scientific), and premixed P/S antibiotics (Nacalai Tesque) in IMDM (Thermo Fisher Scientific) for 7 days, as described previously [15].

\section{Transplantation of SHED-Heps (SHED-HepT) into $\mathrm{CCl}_{4}{ }^{-}$ treated chronic liver fibrosis model mice}

A $\mathrm{CCl}_{4}$ solution in olive oil $(1.0 \mathrm{~mL} / \mathrm{kg}$ body weight; $\mathrm{CCl}_{4}$ to olive oil = 1:4 volume/volume; Wako Pure Chemicals, Osaka, Japan) was intraperitoneally injected into C57BL/6J mice twice a week for 8 weeks, as described previously [12, 19]. Age-matched C57BL/6J mice infused with olive oil (Wako Pure Chemicals) were used as controls. The generated SHED-Heps were washed with 
sterilized with phosphate-buffered saline and kept in cold phosphate-buffered saline (PBS) on ice and quickly infused into the recipient spleen within $10 \mathrm{~min}$ after the preparation to maintain the initially prepared donor cell viability. Four-week-CCl $\mathrm{Cl}_{4}$-treated $\mathrm{C} 57 \mathrm{BL} / 6 \mathrm{~J}$ mice were intrasplenically infused SHED-Heps $\left(1 \times 10^{6} / 10 \mathrm{~g}\right.$ body weight in $100 \mu \mathrm{L}$ of PBS). Age-matched 4-week-CCl ${ }_{4}$ treated C57BL/6J mice were infused with PBS $(100 \mu \mathrm{L})$ as experimental controls. All mice did not receive any immunosuppressants throughout the experiment.

\section{In vivo monitoring of transplanted donor cells}

SHED-Heps were labeled with XenoLight DiR NIR Fluorescent Dye (DiR; $10 \mu \mathrm{g} / \mathrm{mL}$; Perkin Elmer, Waltham, MA) for $30 \mathrm{~min}$ at $37^{\circ} \mathrm{C}$. The DiR-labeled cells or non-labeled SHED-Heps (each $1 \times 10^{6}$ in $100 \mu \mathrm{L}$ of PBS) were intrasplenically infused into 4 -week- $\mathrm{CCl}_{4}$-treated C57BL/6J mice. Ventral images of the mice were obtained $24 \mathrm{~h}$ after infusion with an optical in vivo imaging system IVIS Lumina III (Perkin Elmer) using living image software (Perkin Elmer).

\section{Isolation of whole liver cells (WLCs) from recipient $\mathrm{CCl}_{4^{-}}$ treated mice}

Mouse livers were perfused with Liver Perfusion Medium (Thermo Fisher Scientific) at $37^{\circ} \mathrm{C}$ for $5 \mathrm{~min}$ through the vena cava, followed by $0.1 \%$ collagenase type I (Worthington Biochemicals, Lakewood, NJ) at $37^{\circ} \mathrm{C}$ for $15 \mathrm{~min}$. The liver samples were gently loosened, and WLCs were obtained through a $70-\mu \mathrm{m}$ cell strainer.

\section{Histological assays of mouse liver tissues}

Tissue samples were fixed with formalin and embedded in paraffin. The paraffin sections were used for immunohistochemical and double immunofluorescent analyses, as described in the Additional file 1: Supplementary Methods, previously [11, 14]. The primary antibodies used in immunohistochemical and immunofluorescent assays are summarized in the Additional file 1: Supplementary Table 2. Immunohistochemical and immunofluorescent controls were treated with isotype-matched antibodies instead of primary antibodies. Paraffin sections of normal human and mouse liver tissues were stained with the primary antibodies and examined for cross-reactivity of the primary antibodies. Several paraffin sections were treated with hematoxylin and eosin staining.

\section{In vivo bile assay}

Total bilirubin in mouse serum was measured by colorimetric assay using Bilirubin QuantiChrom Assay Kit (BioAssay Systems, Hayward, CA) according to the manufacturer's instructions. Human albumin (ALB) in mouse serum was analyzed by enzyme-linked immunosorbent assay (ELISA) using human albumin ELISA Quantitation set (Behtyl Laboratory, Montgomery, TX). Feces were collected after fasting overnight. Bile acid in feces was measured by colorimetric assay using Total Bile Acid-Test Wako (Wako Pure Chemical).

\section{In vivo biliary secretion assay}

C57BL/6J mice were intravenously infused with cholyllysyl-fluorescein (CLF; $1 \mathrm{mM}, 100 \mu \mathrm{L}$; Corning, Corning, $\mathrm{NY}$ ) and harvested $2 \mathrm{~h}$ after injection. The amount of CLF in the liver, serum, and urine was quantitated by measuring fluorescence, as described previously [20].

\section{In vitro biliary function assay}

Gene expression of uridine 5'-diphospho-glucuronosyltransferase 1A1 (UGT1A1), membrane metalloendopeptidase (MME), ATP-binding cassette transporter B1 ( $A B C B 1$ ), $A B C 11$, and $A B C C 2$ was analyzed in SHEDHeps by reverse transcription-quantitative polymerase chain reaction (RT-qPCR), as described in the Additional file 1: Supplementary Methods. Distribution of MME, ABCB1, ABCB11, and ABCC2 in SHED-Hep spheroids was analyzed by immunohistochemistry. To analyze hepatobiliary function assays, SHED-Heps were incubated with indirect bilirubin $(25 \mu \mathrm{M}$; Merck, Darmstadt, Germany) in $\mathrm{Ca}^{2+}$-free HBSS (Nacalai Tesque) at $37^{\circ} \mathrm{C}$ for $60 \mathrm{~min}$ and used for determining direct bilirubin by colorimetric assay using QuantiChrom Bilirubin Assay Kit (BioAssay Systems) according to the manufacturer's instructions. SHED-Heps were also incubated with CLF ( $5 \mu \mathrm{M}$; Corning) in $\mathrm{Ca}^{2+}$-free Hank's balanced salt solution (HBSS; Nacalai Tesque) at $37^{\circ} \mathrm{C}$ for $15 \mathrm{~min}$. Intact SHED and human intrahepatic biliary epithelial cells (AXOL, Cambridge, UK) were used as controls.

\section{Immunophenotype analysis of SHED-Heps and WLCs}

The expression of CD90, epithelial cell adhesion molecule (EPCAM), promin-1 (PROM1), MME, CD146, and CD34 were analyzed in SHED-Heps and WLCs by flow cytometric (FCM) analysis, as described in the Additional file 1: Supplementary Methods.

\section{Induction of SHED-Heps into cholangiocyte marker- expressing cells}

SHED-Heps were maintained in William's medium E (Thermo Fisher Scientific) and premixed P/S antibiotics (Nacalai Tasque) supplemented with or without TNFA $(20 \mathrm{ng} / \mathrm{mL}$; PeproTech) for 4 days. Gene expression of human hepatocyte nuclear factor 6 (HNF6), human SRYbox 9 (SOX9), human cytokeratin 7 (KRT7), and human KRT19 was analyzed by RT-qPCR. The distribution of human SOX9, human KRT7, and human ALB was 
analyzed by immunofluorescence, as described in the Additional file 1: Supplementary Methods.

\section{Statistical analysis}

Statistical results were expressed as means \pm standard deviation (SEM) from, at least, triplicate measurements. Comparisons between two groups were analyzed by independent two-tailed Student's $t$ tests. Multiple group comparison was analyzed by one-way repeated measures analysis of variance followed by the Tukey post hoc test. The values of $P<0.05$ were considered statistically significant. All statistical analyses were performed using PRISM 6 software (GraphPad, Software, La Jolla, CA).

\section{Results}

\section{SHED are induced to hepatocyte-lineage committed cells} in vitro

We isolated SHED with a criteria of MSCs, including attached colony formation, cell surface antigen expression, and multipotency into osteoblasts, chondrocytes, and adipocytes [21] (Additional file 1: Supplementary Fig. 1). We cultured SHED under a hepatogenic condition (Additional file 1: Supplementary Fig. 2a). We found a morphological change of spindle-shaped intact SHED into hexagonal-shaped SHED-Heps and bile canaliculilike structures intercellular space of SHED-Heps under the hepatogenic condition (Additional file 1: Supplementary Fig. 2b). RT-qPCR showed that SHED-Heps expressed higher levels for KRT18, ALB, transthyretin (TTR), HNF1A, HNF4A, nuclear receptor subfamily 1 group I member 2 (NR1I2), and peroxisome proliferator activated receptor alpha (PPARA) than intact SHED, but lower in SHED-Heps than in hPHeps; meanwhile, SHED-Heps and intact SHED did not express alpha fetoprotein $(A F P)$, but hPHeps expressed (Additional file 1: Supplementary Fig. 2c). Immunohistochemical analysis detected ALB in SHED-Heps, but not in SHED (Additional file 1: Supplementary Figs. 2d). SHED-Heps showed higher levels of various hepatocyte-specific genes, which are related to urea cycle, glycogen, amino acid, lipid metabolism, xenobiotics, and production of coagulation factor, than intact SHED, but lower than hPHeps by RT-qPCR (Additional file 1: Supplementary Fig. 3).

SHED-Heps exhibited the increased release of glucose, triglyceride, ALB, and fibrinogen, but not AFP into the $\mathrm{CM}$, the decreased ammonia in the $\mathrm{CM}$, the increased intracellular urea, and the enhanced activity of cytochrome P450 3A4 compared to intact SHED (Additional file 1: Supplementary Figs. 4a-4e). SHED-Heps showed the ability of different metabolites storage including indocyanine green uptake and release after $6 \mathrm{~h}$, cytoplasmic accumulation of acetylated low-density lipoprotein uptake, and glycogen storage, but intact SHED did not exhibit any storage function (Additional file 1: Supplementary Figs. $4 \mathrm{f}-4 \mathrm{i})$. The functions were lower in SHED-Heps than in hPHeps (Additional file 1: Supplementary Fig. 4), indicating that SHED-Heps were immature and limited-functional hepatic committed cells.

\section{SHED-HepT ameliorates liver fibrosis in chronically $\mathrm{CCl}_{4}{ }^{-}$ treated mice}

Four-week-CCl $\mathrm{Cl}_{4}$-treated $\mathrm{C} 57 \mathrm{BL} / 6 \mathrm{~J}$ mice were intrasplenically infused SHED-Heps $\left(1 \times 10^{6}\right.$ per mouse $)$ and subsequently received $\mathrm{CCl}_{4}$ for 4 weeks after transplantation (Fig. 1a). Biochemical assays showed the reduced serum levels of aspartate aminotransferase and alanine aminotransferase in recipient mice 4 weeks after transplantation (Additional file 1: Supplementary Fig. 5a). The liver fibrosis was reduced by SHED-HepT, as indicated by the reduced levels of hydroxyproline content, collagen type I mRNA expression, fibrous tissue deposition, and fibrosis score by biochemical analysis, RTqPCR, Masson Trichrome staining, and Ishak scoring (Additional file 1: Supplementary Figs. 5b-5e). Fibrous rates were $0.39 \% \pm 0.63 \%$ (means \pm SEM) in the control non-treated liver, $4.29 \% \pm 2.51 \%$ in the $\mathrm{CCl}_{4}$-treated liver, and $0.91 \% \pm 0.41 \%$ in the SHED-Hep-transplanted $\mathrm{CCl}_{4}$-treated liver 4 weeks after transplantation by Masson Trichrome staining. SHED-HepT showed the decreased levels of alpha-smooth muscle actin 2, smooth muscle, aorta (ACTA2)-positive cells and fibrogenesisrelated marker genes for Acta2, matrix metalloprotease 2 , transforming growth factor beta, and TNFA in the recipient liver by immunohistochemical analysis and RTqPCR (Additional file 1: Supplementary Figs. 5 f, 5 g).

\section{Transplanted donor SHED-Heps integrate in liver tissue of chronically $\mathrm{CCl}_{4}$-treated mice}

In vivo cell tracking analysis showed that fluorescent intensity was detected on the recipient body corresponding to the liver $24 \mathrm{~h}$ after DiR-labeled SHED-HepT, but not on control mouse body (Fig. 1b). FCM analysis showed the expression of a ubiquitous human cell marker, human leukocyte antigens A, B, and C (HLA$\mathrm{ABC}$ ) on WLCs isolated from the recipient mice 4 weeks after transplantation (Fig. 1c). Immunohistochemical analysis using human-specific antibodies showed that HLA-ABC-, human hepatocyte-specific hepatocyte paraffin 1- (HepPar1-), and human ALB-positive cells were detected in the liver parenchymal periphery of recipient mice (Fig. 1d-f). No signal was detected on human and mouse liver tissues by immunohistochemical control tests using isotype-matched antibodies instead of the human-specific antibodies (Additional file 1: Supplementary Fig. 6). Antibody cross-reactivity test evaluated the human specificity of HLA-ABC, HepPar1, and human ALB antibodies, but not the mouse specificity on human 


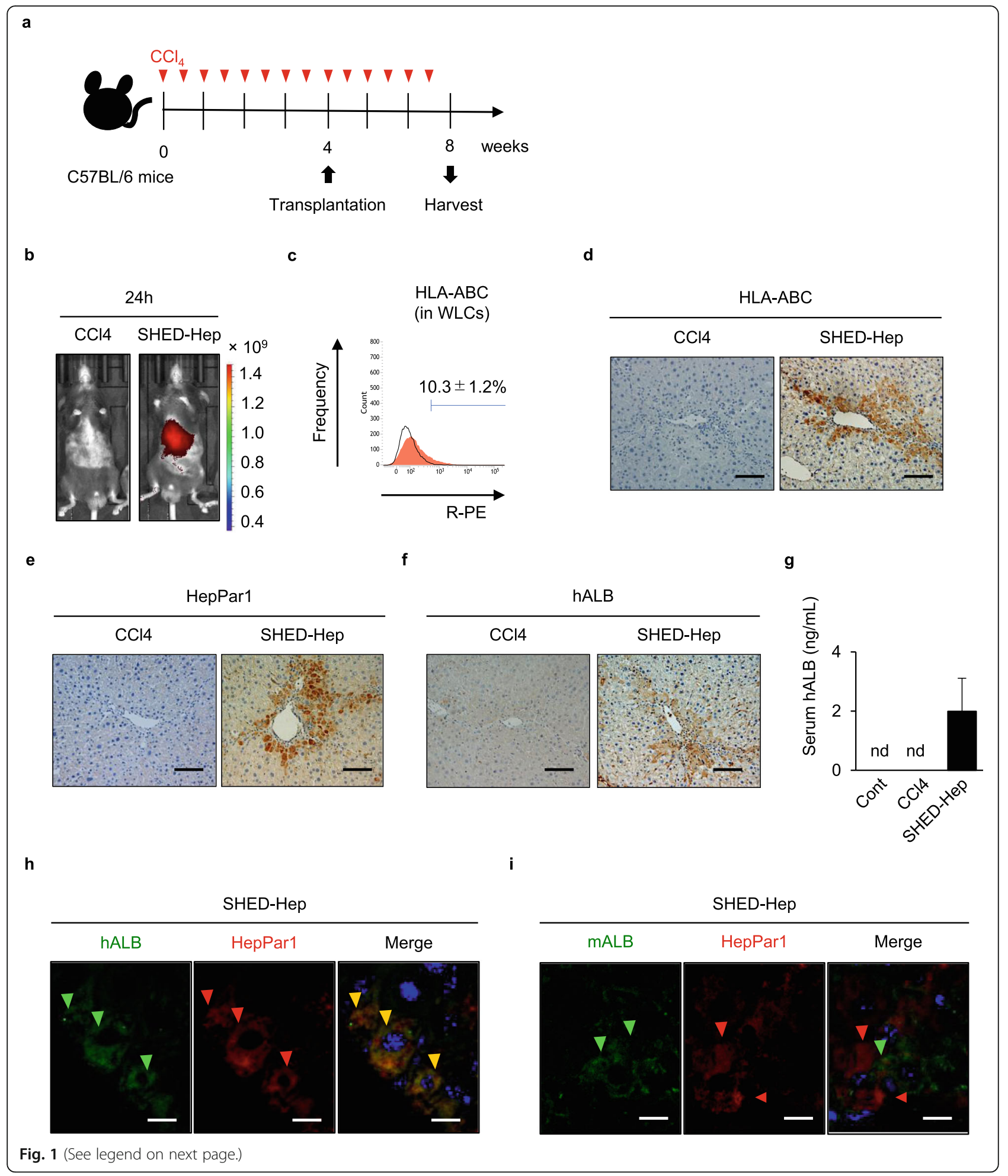




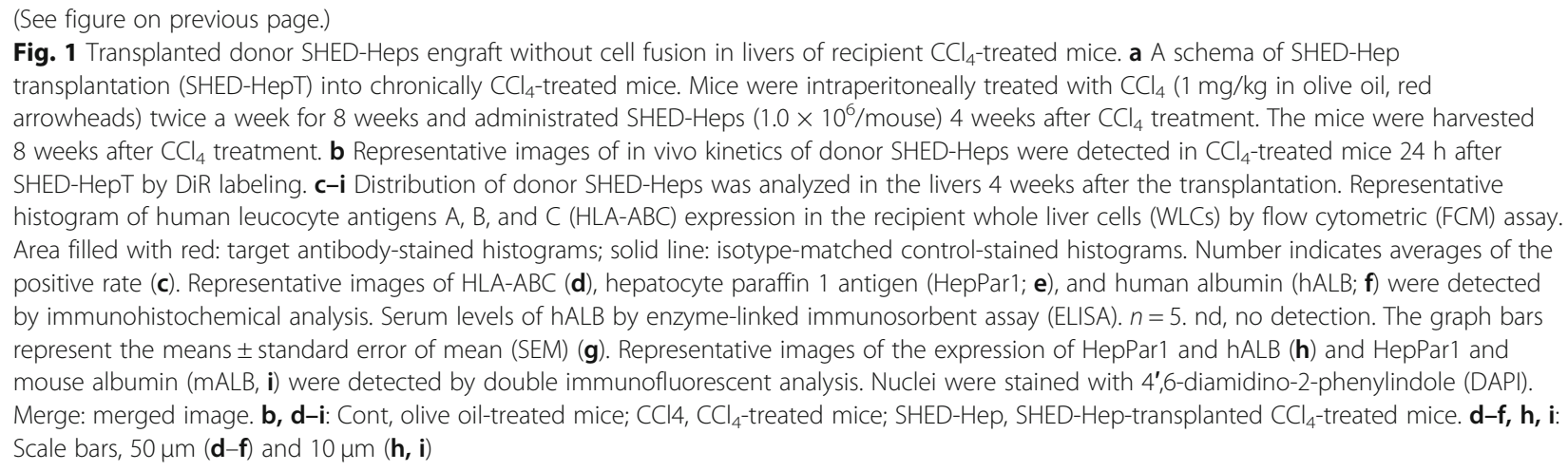

and mouse liver tissues (Additional file 1: Supplementary Figs. $7 \mathrm{a}-7 \mathrm{c}$ ). Homing rates of HepPar1-positive area were $3.31 \% \pm 1.63 \%$ in SHED-Hep-transplanted group, but not detected in both non-treated and $\mathrm{CCl}_{4}$-treated groups. By ELISA, serum human ALB was detected in SHED-Hep-transplanted mice, but not in non-treated and $\mathrm{CCl}_{4}$-treated mice (Fig. 1g). By double immunofluorescent analysis, HepPar1-positive cells co-expressed human ALB, but not mouse ALB, in the liver parenchyma of recipient mice (Fig. 1h, i).

\section{SHED-HepT rescues biliary excretion in chronically $\mathrm{CCl}_{4}$ - treated mice}

$\mathrm{CCl}_{4}$ treatment caused cholestasis in mice, as indicated by the increased levels of serous total bilirubin, the decreased levels of fecal bile acid, and the increased amounts of serous and hepatic CLF by biochemical and biliary excretion analyses (Fig. 2a-c). SHED-HepT recovered the levels of serous total bilirubin and fecal bile acid and the amounts of serous and hepatic CLF 4 weeks after transplantation (Fig. 2a-c). No urinary CLF was found in all experimental groups (Fig. 2c). Histological analysis showed that chronic $\mathrm{CCl}_{4}$ treatment damaged biliary canaliculi and interlobular bile ducts in the parenchymal periphery and periportal region of mouse liver; meanwhile, SHED-HepT regenerated the $\mathrm{CCl}_{4}$-damaged biliary canaliculi and interlobular bile ducts (Fig. 2d).

MME is a pan marker for bile canaliculi. We then demonstrated that the reactivity of MME was reduced in the liver parenchymal periphery of $\mathrm{CCl}_{4}$-treated mice compared to that of control mice by immunohistochemical analysis using anti-human MME antibody (Fig. 2e, Additional file 1: Supplementary Figs. 7d, 8). Interestingly, SHED-HepT increased the MME reactivity in recipient liver (Fig. 2e, Additional file 1: Supplementary Fig. 8). ABCB1, $A B C B 11$, and $A B C C 2$ are bile canaliculispecific hepatobiliary transporters to excrete bile from hetatocytes into bile canaliculi [22-25]. We showed that $\mathrm{ABCB} 1, \mathrm{ABCB} 11$, and $\mathrm{ABCC} 2$ were abundantly expressed in the liver parenchymal periphery of SHEDHep-transplanted $\mathrm{CCl}_{4}$-treated mice compared to that in control $\mathrm{CCl}_{4}$-treated mice by immunohistochemical analysis using the corresponding human-specific antibodies (Additional file 1: Supplementary Fig. 9). Immunohistochemical cross-reactivity and control tests determined that the present anti-ABCC2 antibody showed the human specificity; meanwhile, the anti-ABCB1, antiABCB11, and anti-MME antibodies did not (Additional file 1: Supplementary Figs. 6, 7d, 9). Double immunofluorescent analysis showed that HepPar1-positive cells co-expressed, at least partially, $\mathrm{ABCB} 1, \mathrm{ABCB} 11$, and $\mathrm{ABCC} 2$, as well as MME, in the recipient liver parenchyma (Additional file 1: Supplementary Fig. 10).

Immunohistochemical analysis using anti-human MME antibody showed MME-positive signals in liver interlobular bile ducts of SHED-Hep transplanted mice, but not in those of $\mathrm{CCl}_{4}$-treated mice (Fig. 2f). We then performed immunohistochemical analysis using antimouse KRT19, anti-human KRT19, and anti-human KRT7 antibodies. Mouse KRT19 immunoreactivity was not detected in the liver tissue of $\mathrm{CCl}_{4}$-treated mice; meanwhile, it was found in liver interlobular bile ducts of control mice (Additional file 1: Supplementary Fig. 11a). Human KRT19 and human KRT7 immunoreactivity were detected in liver interlobular bile ducts of SHED-Hep transplanted mice, but not in that of control and $\mathrm{CCl}_{4}$-treated mice (Fig. 2g, h, Additional file 1: Supplementary Figs. 11b, 11c). Immunohistochemical crossreactivity and control tests evaluated human cholangiocyte specificity of anti-human KRT7 and anti-human KRT19 antibodies, as well as mouse cholangiocyte specificity of anti-mouse KRT19 antibody (Additional file 1: Supplementary Fig. 7, 11).

\section{SHED-Heps exhibit cholangiogenic potency under TNFA stimulation in vitro}

SHED-Heps expressed UGT1A1 by RT-qPCR and exhibited the production of direct bilirubin and the function of 


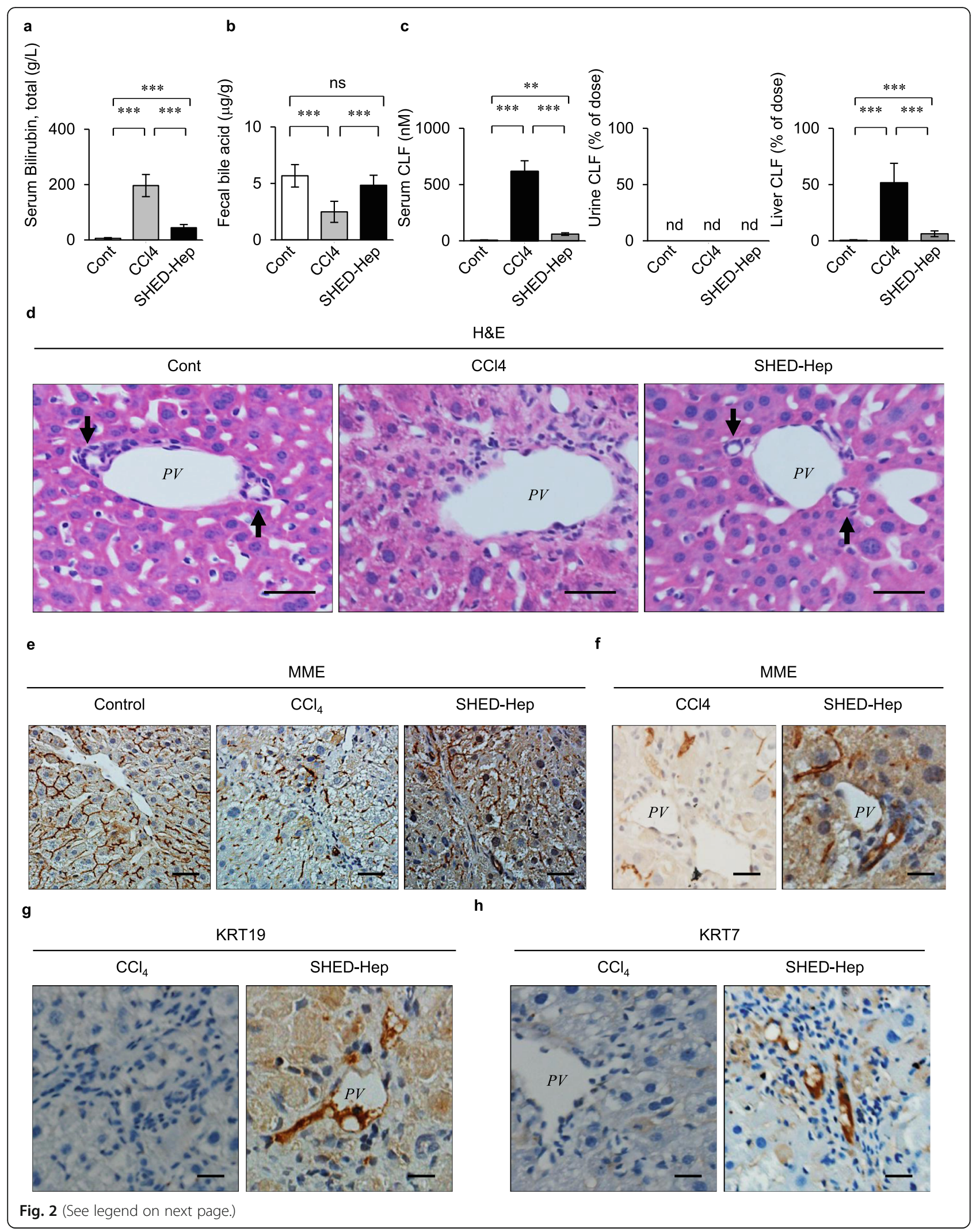


(See figure on previous page.)

Fig. 2 SHED-HepT rescues biliary excretion in recipient $\mathrm{CCl}_{4}$-treated mice. $\mathbf{a}$, b Levels of serum total bilirubin (a) and fecal bile acid (b) 4 weeks after transplantation by colorimetry. $\mathbf{c}$ Cholyl-L-lysyl-fluorescein (CLF) was intravenously infused into mice. The graphs showed the amount of CLF in serum, urine, and liver $2 \mathbf{h}$ after the infusion. $\mathbf{d}$-h Liver tissues were analyzed 4 weeks after SHED-HepT. Representative images of the livers were obtained by hematoxylin and eosin (H\&E) staining (d) and by immunohistochemical analysis using human-specific antibodies to membrane metalloendopeptidase (MME; e, f), keratin 19 (KRT19; g), and KRT7 (KRT7; h). Nuclei were stained with hematoxylin. a-c $n=5$ for all groups. ${ }^{* *} P<$ 0.01 , ${ }^{* *} P<0.005$. nd, no detection; ns, no significance. The graph bars represented the means \pm SEM. $\mathbf{d}$ arrow: bile duct. $\mathbf{d}-\mathbf{h}$ Scale bars, $20 \mu \mathrm{m}$ $(\mathbf{d}, \mathbf{f}-\mathbf{h})$ and $50 \mu \mathrm{m}(\mathbf{e}) . \mathbf{d}, \mathbf{f}-\mathbf{h} P V$, portal vein

hepatobiliary transport by colorimetric assay and CLF staining (Fig. 3a-c). RT-qPCR and immunohistochemical analysis demonstrated the gene and protein expression of hepatobiliary transporters, including MME, ABCB1, ABCB11, and ABCC2 in SHED-Heps (Fig. 3d, e). Meanwhile, intact SHED did not show the hepatobiliary ability (Fig. 3a-e). Further FCM analysis revealed that SHEDHeps expressed hepatic markers, CD90 and MME, but did not express critical hepatic progenitor cells (HPCs) markers, EPCAM, PROM1, and neural cell adhesion molecule 1 (NCAM1) [26, 27] (Fig. 3f). SHED-Heps did not also express CD146, which is considered as a critical and primitive marker for MSCs [28], and definitive hematopoietic marker CD34 by FCM analysis (Fig. 3f). Next, we examined the immunophenotype of WLCs isolated from SHED-Hep-transplanted mice by FCM analysis using human-specific antibodies to CD90, MME, EPCAM, PROM1, NCAM1, CD146, and CD34. Of interest, WLCs expressed human EPCAM, human PROM1, human NCAM1, and human CD146, as well as human CD90 and human MME, but not human CD34 (Fig. 3g), implying that the recipient liver environment may induce donor SHED-Heps into cholangiocytes or cholangiocyteprogenitor cells (CPCs).

TNFA can induce hepatocytes into bile ductular cells [29]. The present and previous [12] studies showed that chronic $\mathrm{CCl}_{4}$ treatment enhances abundant TNFA expression in mouse liver tissue. SHED-Heps were maintained in William's E medium stimulated with or without TNFA for 4 days (Fig. 4a). The culture conditions, especially TNFA-co-stimulating condition, enhanced the expression of HNF6, SOX9, and KRT7 in SHED-Heps by RT-qPCR (Fig. 4b) and the expression of SOX9 and KRT7, but not human ALB, by immunofluorescent analysis (Fig. 4c-e).

\section{Discussion}

Here, we demonstrated that the donor SHED-Heps integrate into liver parenchyma, in recipient chronically $\mathrm{CCl}_{4}$-damaged mice, and regenerate the intrahepatic biliary components. Hepatic damages due to liver fibrosis reduce and lack the distribution of MME in bile canaliculi [30]. Bile canalicular transporters $\mathrm{ABCB} 1, \mathrm{ABCB} 11$, and $\mathrm{ABCC} 2$ are defected in congenital cholestasis, including progressive familial intrahepatic cholestasis type2, benign recurrent intrahepatic cholestasis type2, and Dubin Johnson syndrome [31-33]. HPCs are responsible for developing CPCs and cholangiocytes [26]. Hepatocytes exhibit plasticity to recruit bile ductular cells under liver physiological and pathological conditions [34]. Transplanted hepatocytes can differentiate into bile ductular cells in bile duct ligation model animals [35]. A recent study showed that mature hepatocytes undergo the in situ potency of direct differentiation into SOX9-expressing CPCs through HPCs with mesenchymal phenotype [36]. In this study, WLCs from SHED-Hep-transplanted mice contained SHEDderived HPCs and MSCs, but donor SHED-Heps did not contain HPCs and MSCs. The present study showed that integrated SHED-Heps rescued the expression of MME, $\mathrm{ABCB} 1, \mathrm{ABCB} 11$, and $\mathrm{ABCC} 2$ in bile canaliculi under chronically $\mathrm{CCl}_{4}$-damaged environment. These findings imply a plastic possibility of SHED-Heps to commit to CPCs/cholangiocytes through HPCs in situ and suggest that SHED-Heps might be a source for treating cholestasis associated with bile canaliculi.

Although we revealed that transplanted SHED-Heps recruited bile ductular cells in the recipient livers, the mechanism(s) of SHED-Hep into bile ductular cells remains unknown. Epithelial-mesenchymal transition (EMT) enables mature epithelial cells to undergo transdifferentiation into mesenchymal cells acquired with stem cell-like properties in chronically inflamed tissue [37]. TNFA accelerates EMT via the nuclear factor kappa B-Twist pathway in mammary epithelial cells [38]. Moreover, TNFA can induce the differentiation of hepatocytes into cholangiocytes in organoid culture system [29]. In this study, TNFA enhanced the expression of SOX9 and KRT7 in SHED-Heps. These findings suggest that the inflammatory background by $\mathrm{CCl}_{4}$, such as TNFA, may recruit donor SHED-Heps into CPC/cholangiocyte-like cells through HPC state. Further studies will be required to evaluate the molecular mechanisms in TNFA-triggered cholangiocyte-differentiation of SHED-Hep for treating cholangiopathy. 


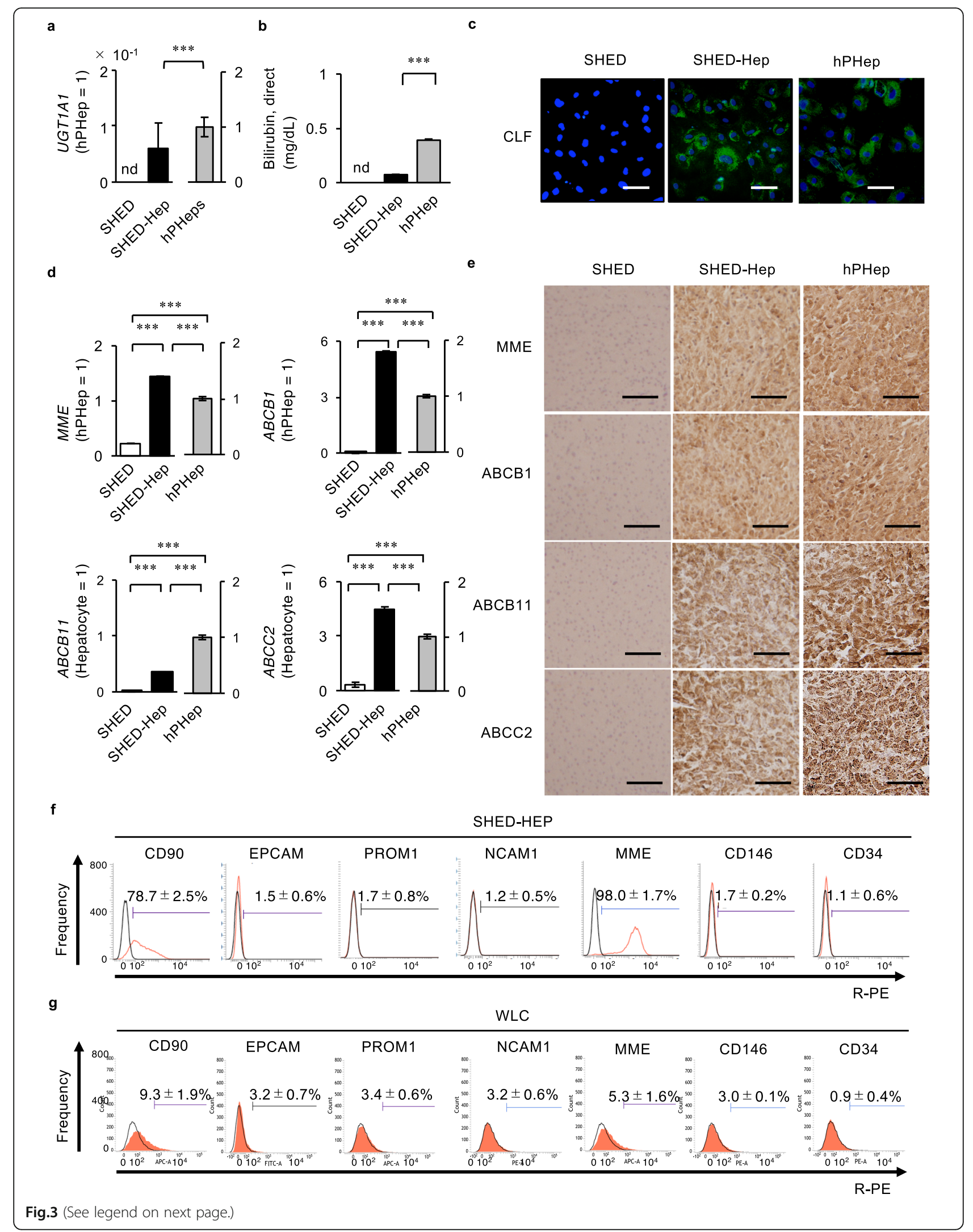




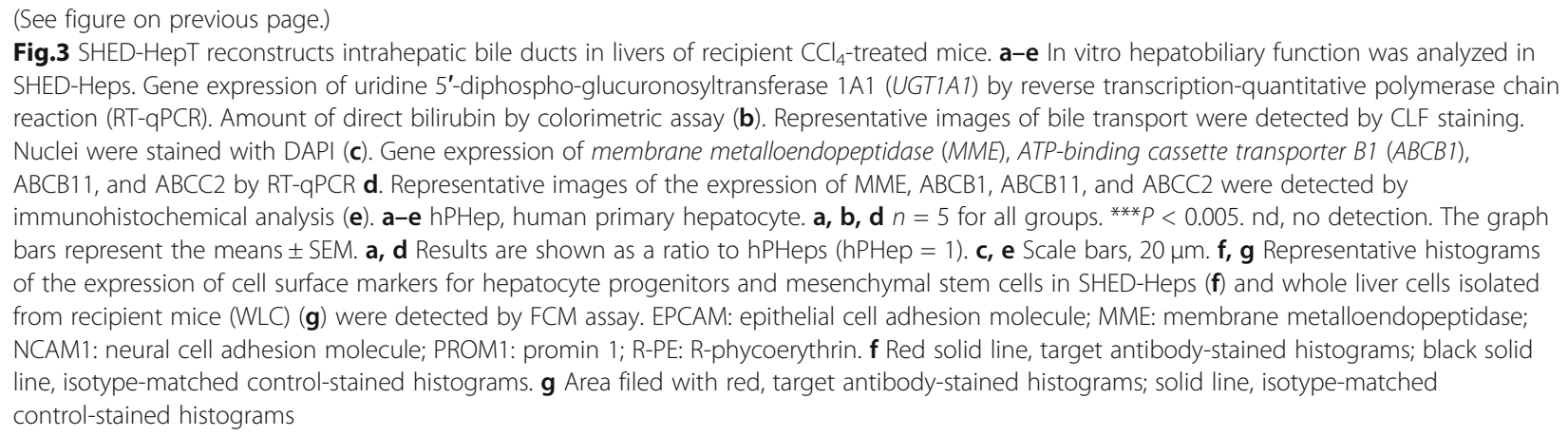

In the present and recent animal studies [12], both systemically transplanted SHED and SHED-Heps recovered chronically CCl4-treated liver fibrosis. Meanwhile, systemically transplanted SHED-Heps, but not SHED, rescued Wilson's disease disorders [14]. Local implantation of SHED-Hep-aggregates gives a benefit for Factor VIII-lacked Hemophilia A [15]. These findings suggest that diverse approaches using SHED and SHED-Heps give an advantage for treating liver disease. A standard operating procedure (SOP) for manufacturing clinicalgrade cell products should be validated before clinical applications [39]. Therefore, currently validated SOP for manufacturing large-scale clinical-grade SHED products [25] might be useful to establish safety-secured and clinical-graded SHED-Hep products. More research will be necessary to improve the quality and safety of SHEDHeps, such as microbial contamination, chromosomal instability, immunogenicity, and tumorigenicity [40]. Post-cryopreserved viability and function of clinicalgrade SHED-Hep products should be needed for transplantation [41], because freeze-thawing process reduces the viability and function of donor hepatocytes due to the damage of mitochondrial respiration [42].

In this study, the long-term survival and engraftment of SHED-Heps were not investigated. In a recent study in Wilsons' disease model animal [14], the long-term survival and engraftment of SHEDHeps are not expected. The reasons why SHED-Heps exhibit the short-term survival and engraftment in the recipient animals are considered due to the immature hepatic differentiation and immunogenicity of donor cells, the xenograft system in immunocompetent animals without any immunosuppressive drug, and the chronical cytotoxic condition after transplantation. On the other hand, SHED-secreting trophic factors including cytokines and extracellular vesicle-containing RNA contents affect immunosuppressive functions [43, 44]. The effect of stanniocalcin 1 secreted from SHED-Heps is anti-hepatitis [14]. Given the results from SHED-Heps transplantation in the intoxicated mice, antiinflammatory effects by SHED-secreting factors may support the regeneration of bile ducts.

In vivo cell fusion with recipient hepatocyte has been considered as a mechanism to integrate donor cells, such as bone marrow hematopoietic cells and hematopoietic cell-derived hepatocytes, in fumarylacetoacetate hydrolase-deficient immunodeficient and chronic $\mathrm{CCl}_{4}$ treated immunocompetent mice [45, 46]. Meanwhile, only bone marrow MSCs can integrated without cell fusion with recipient hepatocytes in the damaged liver [47]. We evaluated that donor SHED can integrate as human HLCs without cell fusion with recipient hepatocytes [12]. Given the present results from fusion failure of donor SHED-Heps, SHED and SHED-Heps may exhibit a unique process to integrate in the recipient damaged liver in vivo. The present hepatic culture system using growth factors and hormones at specific times and in a specific sequence was insufficient to induce SHED into mature and functional HLCs. Based on a recent study, stem cells need to drive them through primitive steak under the stimulation of both inductive and repressive signals for developing into mature and functional hepatocytes [48]. The primitive steak is a critical stage of mimicking developmental biology in an in vitro system, because only the primitive steak can drive definitive endoderm, progenitors, hepatoblasts, and finally mature hepatocytes. Further studies will be necessary to evaluate the in vivo and in vitro mechanism of SHED into mature hepatocytes and cholangiocytes.

\section{Conclusions}

Collectively, SHED-Heps integrate into liver parenchyma, especially its periphery, in recipient chronically $\mathrm{CCl}_{4}$-damaged mice and contributes to the regeneration of intrahepatic bile ducts under fibrosis-associated TNFA microenvironment. Thus, SHED-Heps might be a source for treating cholestasis associated with bile canaliculi. 


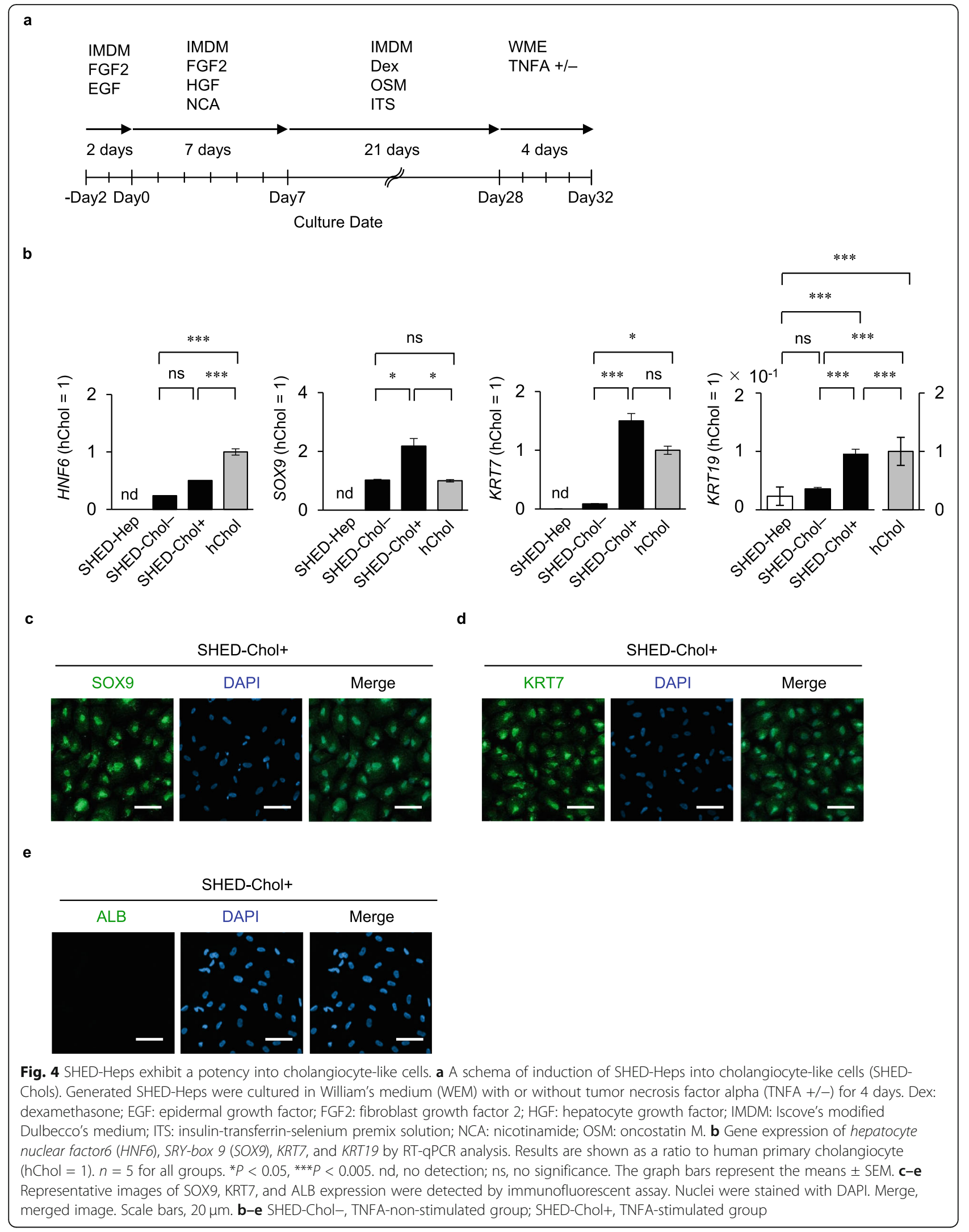




\section{Supplementary Information}

The online version contains supplementary material available at https://doi. org/10.1186/s13287-020-02113-8

Additional file 1. Supplementary Methods. Supplementary References. Supplementary Table 1. The list of specific antibodies used for flow cytometry. Supplementary Table 2. Specific antibodies for immunohistochemistry and immunofluorescence. Supplementary Table 3. List of TaqMan probes for human genes. Supplementary Table 4. List of TaqMan probes for mouse genes. Supplementary Fig. 1. Characterization of stem cells from human exfoliated deciduous teeth (SHED).

Supplementary Fig. 2. Hepatogenic properties of SHED. Supplementary Fig. 3. Expression of hepatic function-associated genes in SHED-Heps. Supplementary Fig. 4. Hepatic functions of SHED-Heps. Supplementary Fig. 5. Effects of SHED-Heps transplantation on liver fibrosis in $\mathrm{CCl}_{4}$ treated mice. Supplementary Fig. 6. Immunohistochemical control tests. Supplementary Fig. 7. Immunohistochemical specificity of antibodies against human leukocyte antigen $A, B$, and $C$ ( $H L A-A B C)$, human hepatocyte paraffin 1 (HepPar1), human ALB, and human MME. Supplementary Fig. 8. Effects of SHED-Heps transplantation on MME expression in liver of $\mathrm{CCl}_{4}$-treated mice. Supplementary Fig. 9. Immunohistochemical localization of biliary transporter markers ATP-binding cassette subfamily B member 1 ( $A B C B 1$ ), ABCB11, and ABCC2. Supplementary Fig. 10. Distribution of biliary canaliculi markers in liver of SHED-Hep-transplanted CCl4-treated mice. Supplementary Fig. 11. Immunohistochemical localization of KRT19 and KRT7.

\section{Abbreviations}

ABCB1: ATP-binding cassette transporter B1; ABCB11: ATP-binding cassette transporter B11; ABCC2: ATP-binding cassette transporter C2; AFP: Alpha fetoprotein; ACTA2: Alpha-smooth muscle actin 2, smooth muscle, aorta; ALB: Albumin; CLF: Cholyl-lysyl-fluorescein; CPCs: Cholangiocyte-progenitor cells; EGF: Epidermal growth factor; ELISA: Enzyme-linked immunosorbent assay; EMT: Epithelial-mesenchymal transition; EPCAM: Epithelial cell adhesion molecule; FCM: Flow cytometry; FGF2: Fibroblast growth factor 2; HBSS: Hank's balanced salt solution; HepPar1: Hepatocyte paraffin 1; HGF: Hepatocyte growth factor; HLA-ABC: Human leukocyte antigens A, B, and $C_{;}$HLCs: Hepatocyte-like cells; HNF1A: Hepatocyte nuclear factor 1A; HNF6: Hepatocyte nuclear factor 6; HPCs: Hepatic progenitor cells; hPHeps: Human primary hepatocytes; IMDM: Iscove's modified Dulbecco's media; ITS: Insulin-transferrin-selenium premix solution; KRT7: Cytokeratin 7; KRT18: Cytokeratin 18; KRT19: Cytokeratin 19; MME: Membrane metalloendopeptidase; MSCs: Mesenchymal stem/stromal cells; NCAM1: Neural cell adhesion molecule 1; NR112: Nuclear receptor subfamily 1 group I member 2; PBS: Phosphate-buffered saline; PPARA: Peroxisome proliferator activated receptor alpha; PROM1: Promin-1; RT-qPCR: Quantitative reverse transcription and polymerase chain reaction; SHED: Stem cells from human exfoliated deciduous teeth; SHED-Heps: SHED-derived hepatocytelike cells; SOP: Standard operating procedure; SOX9: SRY-box 9; TNFA: Tumor necrosis factor alpha; TTR: Transthyretin; UGT1A1: 5'-Diphosphoglucuronosyltransferase $1 \mathrm{~A} 1$; WLCs: Whole liver cells

\section{Acknowledgements}

We would like to thank Editage (www.editage.com) for English language editing. We are very thankful to Ms. Tomoko Yamazaki (Department of Pediatric Surgery, Kyushu University) for her excellent assistance throughout this study. We are thankful to cell innovator (Fukuoka, Japan; www.editage. com) for their helpful microarray data analysis.

\section{Authors' contributions}

R.Y., generation, collection and assembly of data, interpretation of data, statistical analysis; T.Y., conception and design of the study, generation, collection and assembly of data, interpretation of data, statistical analysis, drafting of the manuscript; S.S., K.Y., T.M., H.Y., Y.O., S.O., generation, collection, and assembly of data; interpretation of data; T.T., conception and design, interpretation of data, critical revision of the manuscript, study supervision. All authors approved the final version of the manuscript.

\section{Funding}

This work was supported by the Grants-in-Aid for Scientific Research (A) of the Japan Society for Promotion of Science (JSPS KAKENHI Grant Number $16 \mathrm{H} 02682$ and 25253094 to T.T.)

\section{Availability of data and materials}

All data generated and analyzed during this study are included in this published article and its supplementary information files.

\section{Ethics approval and consent to participate}

Human deciduous teeth were collected from discarded clinical samples from healthy pediatric donors (5-7 years) in the Department of Pediatric Dentistry, Kyushu University Hospital. Human sample handling was approved by Kyushu University Institutional Review Board for Human Genome/Gene Research (Protocol Number: 393-01). We obtained written informed consent from the parents of each patient on behalf of the child donors. All experimental procedures in this study were performed in accordance with the relevant guidelines and regulations.

\section{Consent for publication}

Not applicable.

\section{Competing interests}

The authors declare no competing interests.

\section{Author details}

${ }^{1}$ Department of Pediatric Surgery, Kyushu University Graduate School of Medical Sciences, Fukuoka, Japan. ${ }^{2}$ Department of Anatomy, Faculty of Medicine, Public Health and Nursing, Universitas Gadjah Mada, Yogyakarta, Indonesia. ${ }^{3}$ Department of Molecular Cell Biology and Oral Anatomy, Kyushu University Graduate School of Dental Science, 3-1-1 Maidashi, Higashi-ku, Fukuoka 812-8582, Japan. ${ }^{4}$ Department of Pediatric Dentistry, Kyushu University Graduate School of Dental Science, Fukuoka, Japan. ${ }^{5}$ Department of Anatomic Pathology, Kyushu University Graduate School of Medical Sciences, Fukuoka, Japan. ${ }^{6}$ Department of Pediatrics, Kyushu University Graduate School of Medical Sciences, Fukuoka, Japan. ${ }^{7}$ Fukuoka College of Health Sciences, Fukuoka, Japan.

Received: 28 September 2020 Accepted: 21 December 2020

Published online: 13 January 2021

\section{References}

1. Sze YK, Dhawan A, Taylor RM, Bansal S, Mieli-Vergani G, Rela M, et al. Pediatric liver transplantation for metabolic liver disease: experience at King's College Hospital. Transplantation. 2009;87:87-93.

2. Murray KF, Carithers RL. AASLD practice guidelines: evaluation of the patient for liver transplantation. Hepatology. 2005:41:1407-32.

3. Kim WR, Therneau TM, Benson JT, Kremers WK, Rosen CB, Gores GJ, et al. Deaths on the liver transplant waiting list: an analysis of competing risks. Hepatology. 2006;43:345-51.

4. Fox IJ, Chowdhury JR, Kaufman SS, Goertzen TC, Chowdhury NR, Warkentin $\mathrm{PI}$, et al. Treatment of the Crigler-Najjar syndrome type I with hepatocyte transplantation. N Engl J Med. 1998;338:1422-7.

5. Muraca M, Gerunda G, Neri D, Vilei MT, Granato A, Feltracco P, et al. Hepatocyte transplantation as a treatment for glycogen storage disease type 1a. Lancet. 2002;359:317-8.

6. Dhawan A, Puppi J, Hughes RD, Mitry RR. Human hepatocyte transplantation: current experience and future challenges. Nat Rev Gastroenterol Hepatol. 2010;7:288-98.

7. lansante V, Mitry RR, Filippi C, Fitzpatrick E, Dhawan A. Human hepatocyte transplantation for liver disease: current status and future perspectives. Pediatr Res. 2018;83:232-40.

8. Der Lee K, Kuo TKC, Whang-Peng J, Chung YF, Lin CT, Chou SH, et al. In vitro hepatic differentiation of human mesenchymal stem cells. Hepatology. 2004;40:1275-84.

9. $\mathrm{Hu} C, \mathrm{Wu} Z$, Li L. Pre-treatments enhance the therapeutic effects of mesenchymal stem cells in liver diseases. J Cell Mol Med. 2020;24:40-9.

10. Miura M, Gronthos S, Zhao M, Lu B, Fisher LW, Robey PG, et al. SHED: stem cells from human exfoliated deciduous teeth. Proc Natl Acad Sci U S A. 2003;100:5807-12 
11. Yamaza T, Kentaro A, Chen C, Liu Y, Shi Y, Gronthos S, et al. Immunomodulatory properties of stem cells from human exfoliated deciduous teeth. Stem Cell Res Ther. 2010;1:5.

12. Yamaza T, Alatas FS, Yuniartha R, Yamaza H, Fujiyoshi JK, Yanagi Y, et al. In vivo hepatogenic capacity and therapeutic potential of stem cells from human exfoliated deciduous teeth in liver fibrosis in mice. Stem Cell Res Ther. 2015;6:171

13. Sakai K, Yamamoto A, Matsubara K, Nakamura S, Naruse M, Yamagata M, et al. Human dental pulp-derived stem cells promote locomotor recovery after complete transection of the rat spinal cord by multiple neuroregenerative mechanisms. J Clin Invest. 2012;122:80-90.

14. Fujiyoshi J, Yamaza H, Sonoda S, Yuniartha R, Ihara K, Nonaka K, et al. Therapeutic potential of hepatocyte-like-cells converted from stem cells from human exfoliated deciduous teeth in fulminant Wilson's disease. Sci Rep. 2019;9:1535.

15. Takahashi Y, Yuniartha R, Yamaza T, Sonoda S, Yamaza H, Kirino K, et al. Therapeutic potential of spheroids of stem cells from human exfoliated deciduous teeth for chronic liver fibrosis and hemophilia a. Pediatr Surg Int. 2019:35:1379-88.

16. Desmet $V$, Roskams T, Van Eyken P. Ductular reaction in the liver. Pathol Res Pract. 1995:191:513-24.

17. Alvaro D, Mancino MG, Glaser S, Gaudio E, Marzioni M, Francis $H$, et al Proliferating cholangiocytes: a neuroendocrine compartment in the diseased liver. Gastroenterology. 2007;132:415-31.

18. Clouston AD, Powell EE, Walsh MJ, Richardson MM, Demetris AJ, Jonsson JR. Fibrosis correlates with a ductular reaction in hepatitis C: roles of impaired replication, progenitor cells and steatosis. Hepatology. 2005;41:809-18.

19. Iwanaka T, Yamaza T, Sonoda S, Yoshimaru K, Matsuura T, Yamaza H, et al. A model study for the manufacture and validation of clinical-grade deciduous dental pulp stem cells for chronic liver fibrosis treatment. Stem Cell Res Ther. 2020;11:134.

20. Van Cruchten STJ, De Waart DR, Kunne C, Hooiveld GJEJ, Boekschoten MV, Katan MB, et al. Absorption, distribution, and biliary excretion of cafestol, a potent cholesterol-elevating compound in unfiltered coffees, in mice. Drug Metab Dispos. 2010;38:635-40.

21. Dominici M, Le Blanc K, Mueller I, Slaper-Cortenbach I, Marini FCF, Krause DSDS, et al. Minimal criteria for defining multipotent mesenchymal stromal cells. The International Society for Cellular Therapy position statement. Cytotherapy. 2006:8:315-7

22. Xiao SY, Wang HL, Hart J, Fleming D, Beard MR. Cdna arrays and immunohistochemistry identification of cd10/calla expression in hepatocellular carcinoma. Am J Pathol. 2001;159:1415-21.

23. Nies AT, Keppler D. The apical conjugate efflux pump ABCC2 (MRP2). Pflugers Arch Eur J Physiol. 2007;453:643-59.

24. Gissen P, Arias IM. Structural and functional hepatocyte polarity and liver disease. J Hepatol. 2015;63:1023-37.

25. Lagana SM, Salomao M, Remotti HE, Knisely AS, Moreira RK. Bile salt export pump: a sensitive and specific immunohistochemical marker of hepatocellular carcinoma. Histopathology. 2015;66:598-602.

26. Turner R, Lozoya O, Wang Y, Cardinale V, Gaudio E, Alpini G, et al. Human hepatic stem cell and maturational liver lineage biology. Hepatology. 2011 53:1035-45.

27. Chaudhari $P$, Tian L, Deshmukh A, Jang YY. Expression kinetics of hepatic progenitor markers in cellular models of human liver development recapitulating hepatocyte and biliary cell fate commitment. Exp Biol Med. 2016;241:1653-62.

28. Sacchetti B, Funari A, Michienzi S, Di Cesare S, Piersanti S, Saggio I, et al. Self-renewing osteoprogenitors in bone marrow sinusoids can organize a hematopoietic microenvironment. Cell. 2007;131:324-36.

29. Nishikawa Y, Sone M, Nagahama Y, Kumagai E, Doi Y, Omori Y, et al. Tumor necrosis factor-a promotes bile ductular transdifferentiation of mature rat hepatocytes in vitro. J Cell Biochem. 2013;114:831-43.

30. Shousha S, Gadir F, Peston D, Bansi D, Thillainaygam AV, Murray-Lyon IM. CD10 immunostaining of bile canaliculi in liver biopsies: change of staining pattern with the development of cirrhosis. Histopathology. 2004;45:335-42.

31. Zollner G. Hepatobiliary transporter expression in percutaneous liver biopsies of patients with cholestatic liver diseases. Hepatology. 2001;33:63346.

32. Meier Y, Pauli-Magnus C, Zanger UM, Klein K, Schaeffeler E, Nussler AK, et al. Interindividual variability of canalicular ATP-binding-cassette (ABC)transporter expression in human liver. Hepatology. 2006;44:62-74.
33. Stapelbroek JM, van Erpecum KJ, Klomp LWJ, Houwen RHJ. Liver disease associated with canalicular transport defects: current and future therapies. J Hepatol. 2010;52:258-71.

34. Li W, Li L, Hui L. Cell plasticity in liver regeneration. Trends Cell Biol. 2020;30: 329-38.

35. Yovchev MI, Locker J, Oertel M. Biliary fibrosis drives liver repopulation and phenotype transition of transplanted hepatocytes. J Hepatol. 2016;64:134857.

36. Tarlow BD, Pelz C, Naugler WE, Wakefield L, Wilson EM, Finegold MJ, et al. Bipotential adult liver progenitors are derived from chronically injured mature hepatocytes. Cell Stem Cell. 2014;15:605-18.

37. Kalluri R, Weinberg RA. The basics of epithelial-mesenchymal transition. J Clin Invest. 2009;119:1420-8.

38. Li CW, Xia W, Huo L, Lim SO, Wu Y, Hsu JL, et al. Epithelial-mesenchymal transition induced by TNF-a requires NF-kB-mediated transcriptional upregulation of Twist1. Cancer Res. 2012;72:1290-300.

39. Unger C, Skottman H, Blomberg P. Sirac dilber M, Hovatta O. good manufacturing practice and clinical-grade human embryonic stem cell lines. Hum Mol Genet. 2008;17:R48-53.

40. Wang Y, Han ZB, Song YP, Han ZC. Safety of mesenchymal stem cells for clinical application. Stem Cells Int. 2012;652034.

41. Gramignoli R, Tahan V, Dorko K, Venkataramanan R, Fox IJ, Ellis ECS, et al. Rapid and sensitive assessment of human hepatocyte functions. Cell Transplant. 2014;23:1545-56.

42. Stéphenne X, Najimi M, Dung KN, Smets F, Hue L, Guigas B, et al. Cryopreservation of human hepatocytes alters the mitochondrial respiratory chain complex 1. Cell Transplant. 2007;16:409-19.

43. Hirata $M$, Ishigami M, Matsushita $Y$, Ito T, Hattori $H$, Hibi $H$, et al. Multifaceted therapeutic benefits of factors derived from dental pulp stem cells for mouse liver fibrosis. Stem Cells Transl Med. 2016;5:1416-24.

44. Sonoda S, Murata S, Nishida K, Kato H, Uehara N, Kyumoto-Nakamura Y, et al. Extracellular vesicles from deciduous pulp stem cells recover bone loss by regulating telomerase activity in an osteoporosis mouse model. Stem Cell Res Ther. 2020;11:296.

45. Wang $X$, Willenbring $H$, Akkari $Y$, Torimaru $Y$, Foster $M$, Al-Dhalimy $M$, et al, Cell fusion is the principal source of bone-marrow-derived hepatocytes. Nature. 2003:422:897-901.

46. Quintana-Bustamante O, Alvarez-Barrientos A, Kofman AV, Fabregat I, Bueren JA, Theise ND, et al. Hematopoietic mobilization in mice increases the presence of bone marrow-derived hepatocytes via in vivo cell fusion. Hepatology. 2006;43:108-16.

47. Sato $Y$, Araki H, Kato J, Nakamura K, Kawano Y, Kobune M, et al. Human mesenchymal stem cells xenografted directly to rat liver are differentiated into human hepatocytes without fusion. Blood. 2005;106:756-63.

48. Ang LT, Tan AKY, Autio Ml, Goh SH, Choo SH, Lee KL, et al. A roadmap for human liver differentiation from pluripotent stem cells. Cell Rep. 2018;22: 2190-205.

\section{Publisher's Note}

Springer Nature remains neutral with regard to jurisdictional claims in published maps and institutional affiliations.

Ready to submit your research? Choose BMC and benefit from:

- fast, convenient online submission

- thorough peer review by experienced researchers in your field

- rapid publication on acceptance

- support for research data, including large and complex data types

- gold Open Access which fosters wider collaboration and increased citations

- maximum visibility for your research: over $100 \mathrm{M}$ website views per year

At $\mathrm{BMC}$, research is always in progress.

Learn more biomedcentral.com/submissions 